\title{
Unusual magnetic resonance imaging findings in a patient with posterior reversible encephalopathy syndrome
}

\author{
Hyug-Gi Kim ${ }^{1}$, Kyung Mi Lee ${ }^{1}$, Jin San Lee ${ }^{2}$ \\ ${ }^{1}$ Department of Radiology, ${ }^{2}$ Department of Neurology, Kyung Hee University Hospital, Kyung Hee University College of Medicine, Seoul, Korea \\ Correspondence to: Jin San Lee, MD, PhD. Department of Neurology, Kyung Hee University Hospital, 23, Kyungheedae-ro, Dondaemun-gu, Seoul \\ 02447, Korea. Email: xpist@naver.com.
}

Submitted Oct 11, 2018. Accepted for publication Oct 25, 2018.

doi: 10.21037/qims.2018.10.10

View this article at: http://dx.doi.org/10.21037/qims.2018.10.10

A 37-year-old male presented to the neurology department after developing sudden headache, nausea, and visual disturbances. The patient had been diagnosed with hypertension 5 years previously, but was not currently taking appropriate medication; he also had a 20-pack/year history of smoking. At the time of admission, the patient's blood pressure was $210 / 100 \mathrm{mmHg}$ and remained high under continuous monitoring during the first $24 \mathrm{~h}$. Two days later, he noted transient left leg weakness lasting approximately $2-3 \mathrm{~min}$. Brain magnetic resonance imaging (MRI) revealed multiple bilateral hyperintense subcortical white matter lesions in the posterior circulation and the middle cerebral artery territories including the parieto-occipital lobes, temporal pole, and pons, on fluid-attenuated inversion recovery (FLAIR) sequence (Figure 1A). In addition, a focal hyperintense lesion at the right centrum semiovale was observed on diffusion weighted imaging and the lesion was confirmed as an acute ischemic cerebral infarction through low signal intensity on apparent diffusion coefficient map (Figure 1B). Magnetic resonance angiography of the brain revealed a saccular aneurysm $5 \mathrm{~mm}$ in diameter at the origin of the right middle cerebral artery bifurcation (Figure 1C). Several cerebral microbleeds in the left basal ganglia, bilateral occipital lobes, and pons were observed on susceptibility-weighted imaging (Figure 1D). The patient received antihypertensive treatment, and blood pressure was satisfactorily controlled within $7 \mathrm{~d}$; his symptoms of headache, nausea, and visual disturbances also noticeably improved. Follow-up brain MRI was performed 4 months later and revealed near complete resolution of bilateral hyperintense lesions in the posterior circulation and the middle cerebral artery territories on FLAIR sequence (Figure 1E). We finally diagnosed the patient with posterior reversible encephalopathy syndrome (PRES).

PRES is an acute neurological syndrome clinically characterized by the onset of headache, seizure, visual disturbances, altered mental status, or focal neurological deficits; it is considered to be triggered by the elevation of blood pressure beyond autoregulation (1). Previous studies have suggested that a sudden elevation of blood pressure produces a breakdown in autoregulation of cerebral vessels, causing extravasation of fluid into the surrounding brain tissue and inducing reversible vasogenic edema in patients with PRES (2,3). Typical and atypical MRI findings of PRES have been reported and mainly involve the supratentorial white matter in the parieto-occipital lobes, brain stem, and cerebellum. However, our case differs from those in previous reports in several aspects, including both atypical location and atypical imaging findings.

First, the lesions in patients with typical PRES are located primarily in the bilateral parieto-occipital regions $(>90 \%$ of cases), followed by the high posterior frontal, temporal, cerebellar, basal ganglia, and brainstem regions (4). However, FLAIR-MRI of this patient revealed extensive white matter hyperintensities in the parieto-occipital regions and pons, as well as in the temporal pole. The deep white matter in the temporal pole is fed mainly by the medullary arteries originating from the middle cerebral artery. The medullary arteries are vulnerable to impaired contractility under prolonged uncontrolled hypertension (5). This involvement pattern is not common in patients with PRES, and there is only one published report describing 

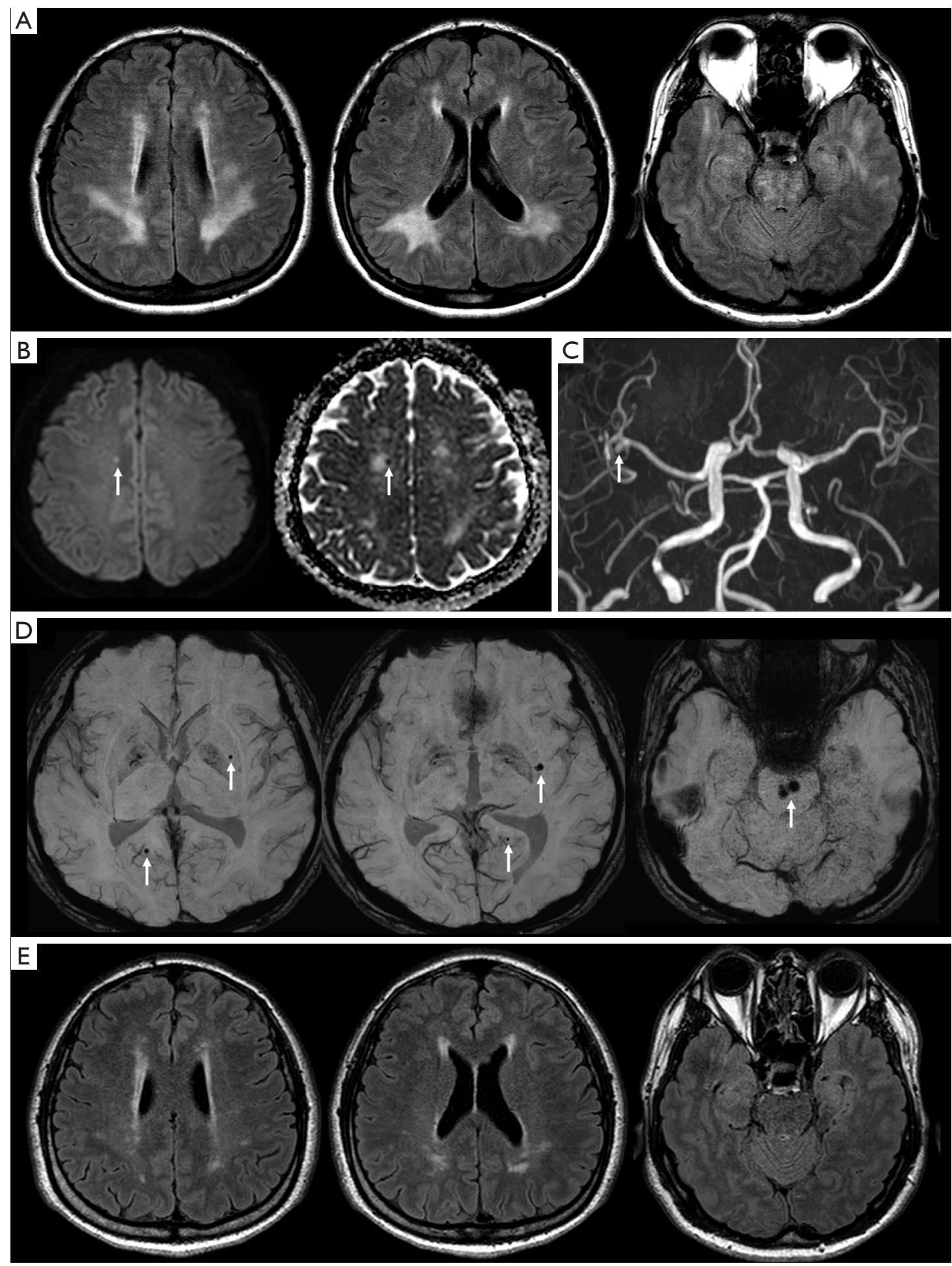

Figure 1 MRI findings of the patient. (A) Multiple bilateral hyperintense subcortical white matter lesions in the bilateral parieto-occipital lobes, temporal pole white matter, and pons on fluid-attenuated inversion recovery sequence; (B) an acute focal infarction at the right centrum semiovale based on diffusion weighted imaging and apparent diffusion coefficient map (arrows); (C) a saccular aneurysm at the origin of the right middle cerebral artery bifurcation (arrow); (D) several cerebral microbleeds in the left basal ganglia, bilateral occipital lobes, and pons on susceptibility-weighted imaging (arrows); (E) follow-up brain MRI. MRI, magnetic resonance imaging. 
the involvement of temporal pole white matter in a patient with PRES (6). Second, there have been no reported cases of acute cerebral infarction, aneurysm, and microbleeds in a single patient with PRES. Acute hypertension can cause cerebral vasculature vasospasm, followed by a decrease in blood flow and intravascular thrombosis. These factors appear to cause ischemic infarction and cytotoxic edema (7). Sustained high blood pressure for a long time also can cause cerebral microbleeds and aneurysm. Alternatively, the patient's smoking history may have contributed to the formation of cerebral aneurysm. Considering that PRES is characterized by reversible subcortical vasogenic edema predominant in the posterior brain regions (frequently, in the brain stem, basal ganglia, and/or cerebellum) (8), it is noteworthy that this patient exhibited the spatial coincidence of vasogenic edema with cerebral microbleeds.

We consider that acute and chronic uncontrolled hypertension led to PRES, acute cerebral infarction, cerebral aneurysm, and microbleeds in this patient. We herein report a case of PRES with various abnormal MRI findings and suggest that PRES is a radiologically heterogeneous syndrome.

\section{Acknowledgements}

Funding: This work was supported by a grant from Basic Science Research Program through the Ministry of Education of the Republic of Korea (NRF2016R1D1A1B03933173 and 2018R1D1A1B07041308).

\section{Footnote}

Conflicts of Interest: The authors have no conflicts of interest to declare.

Ethical Statement: The Institutional Review Board (IRB) of our hospital waived IRB approval because our single

Cite this article as: Kim HG, Lee KM, Lee JS. Unusual magnetic resonance imaging findings in a patient with posterior reversible encephalopathy syndrome. Quant Imaging Med Surg 2018;8(10):1066-1068. doi: 10.21037/qims.2018.10.10 case report did not perform any prospective intervention. Written informed consent was obtained from the patient.

\section{References}

1. Bartynski WS. Posterior reversible encephalopathy syndrome, part 2: controversies surrounding pathophysiology of vasogenic edema. AJNR Am J Neuroradiol 2008;29:1043-9.

2. Sundgren PC, Edvardsson B, Holtås S. Serial investigation of perfusion disturbances and vasogenic oedema in hypertensive encephalopathy by diffusion and perfusion weighted imaging. Neuroradiology 2002;44:299-304.

3. Chu K, Kang DW, Lee SH, Kim M. Diffusionweighted MR findings in brain stem hypertensive encephalopathy: a possibility of cytotoxic edema? Eur Neurol 2001;46:220-2.

4. Gao B, Lyu C, Lerner A, McKinney AM. Controversy of posterior reversible encephalopathy syndrome: what have we learnt in the last 20 years? J Neurol Neurosurg Psychiatry 2018;89:14-20.

5. Pantoni L. Cerebral small vessel disease: from pathogenesis and clinical characteristics to therapeutic challenges. Lancet Neurol 2010;9:689-701.

6. Ohira J, Mori N, Kajikawa S, Nakamura T, Arisato T, Takahashi M. Posterior Reversible Encephalopathy Syndrome with Extensive Deep White Matter Lesions Including the Temporal Pole. Intern Med 2016;55:3529-33.

7. Schwartz RB, Feske SK, Polak JF, DeGirolami U, Iaia A, Beckner KM, Bravo SM, Klufas RA, Chai RY, Repke JT. Preeclampsia-eclampsia: clinical and neuroradiographic correlates and insights into the pathogenesis of hypertensive encephalopathy. Radiology 2000;217:371-6.

8. Renard D. Cerebral microbleeds: a magnetic resonance imaging review of common and less common causes. Eur J Neurol 2018;25:441-50. 